Bull. Korean Math. Soc. 46 (2009), No. 5, pp. 867-871

DOI 10.4134/BKMS.2009.46.5.867

\title{
INDEPENDENTLY GENERATED MODULES
}

\author{
Muhammet Tamer Koşan and Tufan Özdin
}

\begin{abstract}
A module $M$ over a ring $R$ is said to satisfy $(P)$ if every generating set of $M$ contains an independent generating set. The following results are proved;

(1) Let $\tau=\left(\mathbb{T}_{\tau}, \mathbb{F}_{\tau}\right)$ be a hereditary torsion theory such that $\mathbb{T}_{\tau} \neq$ Mod- $R$. Then every $\tau$-torsionfree $R$-module satisfies $(P)$ if and only if $S=R / \tau(R)$ is a division ring.

(2) Let $\mathcal{K}$ be a hereditary pre-torsion class of modules. Then every module in $\mathcal{K}$ satisfies $(P)$ if and only if either $\mathcal{K}=\{0\}$ or $S=R / \operatorname{Soc}_{\mathcal{K}}(R)$ is a division ring, where $\operatorname{Soc}_{\mathcal{K}}(R)=\cap\left\{I \leq R_{R}: R / I \in \mathcal{K}\right\}$.
\end{abstract}

For a right $R$-module $M$, a subset $X$ of $M$ is said to be a generating set of $M$ if $M=\Sigma_{x \in X} x R$; and a minimal generating set of $M$ is any generating set $Y$ of $M$ such that no proper subset of $Y$ can generate $M$. A generating set $X$ of $M$ is called an independent generating set if $\Sigma_{x \in X} x R=\oplus_{x \in X} x R$. Clearly, every independent generating set of $M$ is a minimal generating set, but the converse is not true in general. For example, the set $\{2,3\}$ is a minimal generating set of $\mathbb{Z}_{\mathbb{Z}}$ but not an independent generating set.

It is well-known that every generating set of a right vector space over a division ring contains a minimal generating set (or a basis). This motivated various interests in characterizing the rings $R$ such that every module in a certain class of right $R$-modules contains a minimal generating set, or every generating set of each module in a certain class of right $R$-modules contains a minimal generating set (see, for example, [2], [8], [9], [11]).

In [2, Theorem 2.3], the authors proved that $R$ is a division ring if and only if every $R$-module has a basis if and only if every irredundant subset of an $R$-module is independent. This result can be considered in a more general context of a torsion theory. For an $R$-module $M, M$ is said to satisfy $(P)$ if every

Received July 13, 2008

2000 Mathematics Subject Classification. 16D10.

Key words and phrases. generated set for modules, basis, (non)-singular modules, division ring, torsion theory.

The content of this paper is a part of a thesis written by Tufan Özdin under the supervision of M. Tamer Koşan (Gebze Institute of Technology).

(C)2009 The Korean Mathematical Society 
generating set of $M$ contains an independent generating set. For a hereditary torsion theory $\tau=\left(\mathbb{T}_{\tau}, \mathbb{F}_{\tau}\right)$, the paper is concerned with the following questions:

(1) When does every $\tau$-torsion module satisfy $(P)$ ?

(2) When does every $\tau$-torsionfree module satisfy $(P)$ ?

Throughout this paper $R$ denotes an associative ring with unit and $M$ is a right unitary $R$-module. For a module $M$, the notions " $\leq$ ", "Soc $(M)$ ", " $x^{\perp}$ ", and " $Z(M)$ " denote the submodule, the socle, the right annihilator of an element $x$, and the singular submodule of $M$, respectively. Moreover, $Z_{2}(M)$ is defined by $Z(M / Z(M))=Z_{2}(M) / Z(M)$. If $M=Z_{2}(M)$, we say that $M$ is Goldie torsion. If $Z(M)=0$, then $M$ is called nonsingular. A module is called quasi-cyclic if each of its finitely generated submodules is contained in a cyclic submodule (see [7]). According to Bass [3], for a sequence $\left\{a_{n}: n=1,2, \ldots\right\}$ of elements of $R$, let $F$ the free $R$-module with basis $x_{1}, x_{2}, \ldots, G$ the submodule of $F$ generated by the set $\left\{x_{n}-a_{n} x_{n+1}: n=1,2, \ldots\right\}$, and $\left[F,\left\{a_{n}\right\}, G\right]$ the quotient module $F / G$. Note that $\left[F,\left\{a_{n}\right\}, G\right]$ is a quasi-cyclic module.

We will refer to [1], [4] and [6] for all undefined notions used in the text.

We begin with the following easy but useful lemma.

Lemma 1. Let $x R, y R$ be nonzero cyclic $R$-modules with $x^{\perp} \neq y^{\perp}$, and let $M=x R \oplus x R \oplus y R \oplus y R$. Then there is a submodule $N$ of $M$ such that $N$ does not satisfy $(P)$.

Proof. Without loss of generality, we may assume that $x^{\perp} \nsubseteq y^{\perp}$. Let $u=$ $(x, x, y, 0)$ and $v=(0, x, y, 0)$, and let $N$ be the submodule of $M$ generated by $\{u, v\}$. Since $u \notin v R$ and $v \notin u R,\{u, v\}$ is a minimal generating set of $N$. But $\{u, v\}$ is not an independent generating set of $N$ since $0 \neq\left(0,0, y x^{\perp}, 0\right) \subseteq$ $u R \cap v R$. Therefore, the generating set $\{u, v\}$ does not contain any independent generating sets of $N$.

Theorem 2. Let $\tau=\left(\mathbb{T}_{\tau}, \mathbb{F}_{\tau}\right)$ be a hereditary torsion theory such that $\mathbb{T}_{\tau} \neq$ Mod-R. The following are equivalent for a ring $R$ :

(1) Every $R$-module satisfies $(P)$.

(2) $\tau(R)=0$ and every $\tau$-torsionfree module satisfies $(P)$.

(3) $R$ is a division ring.

Proof. $(3) \Rightarrow(1)$. It is well-known.

$(1) \Rightarrow(2)$. Suppose $0 \neq a \in \tau(R)$. If $a^{\perp}=0$, then $R_{R} \cong a R \in \mathbb{T}_{\tau}$. Thus, $R \in T_{\tau}$, implying Mod- $R=\mathbb{T}_{\tau}$. This contradicts the assumption on $\tau$. Hence, $a b=0$ for some $0 \neq b \in R$. Therefore, by Lemma 1 , the module $a R \oplus a R \oplus R \oplus R$ has a submodule $N$ such that $N$ does not satisfies $(P)$. This contradiction shows that $\tau\left(R_{R}\right)=0$.

$(2) \Rightarrow(3)$. Suppose $R$ satisfies (2). First we claim that, for any $\tau$-torsionfree module $M$ with $x \in M$ and $r \in R, x r=0$ implies that $x=0$ or $r=0$. 
For, if not, by Lemma 1 , the $\tau$-torsionfree module $x R \oplus x R \oplus R \oplus R$ has a submodule $N$ such that $N$ does not satisfies $(P)$. This is a contradiction. In particular, our claim implies that $R$ is a domain. Suppose $R$ is not a division ring. Then $a R \neq R$ for some $0 \neq a \in R$. Let $a_{n}=a$ for $n=1,2, \ldots$, let $F$ be the free $R$-module with basis $\left\{x_{n}: n=1,2, \ldots\right\}$, and $G$ the submodule of $F$ generated by the set $\left\{x_{n}-x_{n+1} a_{n}: n=1,2, \ldots\right\}$. Set $H=F / G$. If $\overline{x_{1}}=x_{1}+G \in \tau\left(H_{R}\right)$, then, since $R$ is not in $\mathbb{T}_{\tau}, \overline{x_{1}} c=\overline{0}$ for some nonzero element $c \in R$. But it is straightforward to check that this is impossible. Therefore, $\overline{x_{1}} \notin \tau(H)$. So $H / \tau(H)$ is a nonzero $\tau$-torsionfree module. Note that $\left\{\overline{x_{n}}+\tau(H): n=1,2, \ldots\right\}$ is a generating set of $H / \tau(H)$. By (2), there is a nonempty set $\mathbf{L}$ of positive integers such that $\left\{\overline{x_{n}}+\tau(H): n \in \mathbf{L}\right\}$ is an independent generating set of $H / \tau(H)$. Let $m$ be the least number in $\mathbf{L}$. Note that $\overline{x_{m}}+\tau(H)=\left[\overline{x_{m+k}}+\tau(H)\right] a^{k}$ for $k=1,2, \ldots$ It must be $\mathbf{L}=\{m\}$, i.e., $H / \tau(H)$ is generated by $\overline{x_{m}}+\tau(H)$. Therefore, $\overline{x_{m+1}}+\tau(H)=\left[\overline{x_{m}}+\tau(H)\right] r$ for some $r \in R$, i.e., $\left[\overline{x_{m+1}}+\tau(H)\right](1-a r)=\overline{\overline{0}}(=\overline{0}+\tau(H))$. Now by the claim above, $\overline{x_{m+1}}+\tau(H)=\overline{\overline{0}}$ or $1-a r=0$. Since $\overline{x_{1}} \notin \tau(H)$, we have $\overline{x_{m+1}} \notin \tau(H)$, and thus $1-a r=0$, i.e., $a R=R$. This is a contradiction.

Applying Theorem 2 to the Goldie torsion theory $\tau$ yields the next corollary.

Corollary 3. The ring $R$ is a division ring if and only if $R$ is right non-singular and every non-singular $R$-module satisfies $(P)$.

Let $S=R / \tau(R)$ be the factor ring and $\gamma: R \longrightarrow S$ be the canonical ring homomorphism. Then $\gamma$ induces a hereditary torsion theory $\sigma=\gamma_{\#}(\tau)$ on Mod- $S$ defined by the condition that an $S$-module $N$ is a $\sigma$-torsion $S$-module if and only if $N_{R}$ is a $\tau$-torsion module (see [5, p. 433]).

Theorem 4. Let $\tau=\left(\mathbb{T}_{\tau}, \mathbb{F}_{\tau}\right)$ be a hereditary torsion theory such that $\mathbb{T}_{\tau} \neq$ Mod-R. Then every $\tau$-torsionfree $R$-module satisfies $(P)$ if and only if $S=$ $R / \tau(R)$ is a division ring.

Proof. " $\Rightarrow "$. Since $R$ is not in $\mathbb{T}_{\tau}$ and $\tau(R / \tau(R))=0, S$ is nonzero and $\sigma(S)=0$. Let $N_{S}$ be a $\sigma$-torsionfree module with a generating set $Y$. Then $N$ is a $\tau$-torsionfree $R$-module with a generating set $Y$. By the assumption, $N_{R}=\oplus_{x \in X} x R$ for a subset $X$ of $Y$. It follows that $N_{S}=\oplus_{x \in X} x S$. By Theorem $1, S$ is a division ring.

" $\Leftarrow$ ". Let $N$ be a $\tau$-torsionfree $R$-module with a generating set $Y$. Since $N \tau(R) \subseteq \tau(N)$, we see $N \tau(R)=0$. Thus, $N$ is an $S$-module and hence is a $\sigma$-torsionfree module with a generating set $Y$. Since $S$ is a division ring, by Theorem 2, we have $N_{S}=\oplus_{x \in X} x S$ for a subset $X$ of $Y$. Thus, $N_{R}=$ $\oplus_{x \in X} x R$.

When $\tau$ is the Goldie torsion theory, Theorem 4 gives the next consequence.

Corollary 5. Every nonsingular $R$-module satisfies $(P)$ if and only if $R=$ $Z_{2}(R)$ or $R / Z_{2}(R)$ is a division ring. 
Let $\mathcal{K}$ be a hereditary pre-torsion class of modules and $\operatorname{Soc}_{\mathcal{K}}(R)=\cap\{I: I \in$ $\left.H_{\mathcal{K}}(R)\right\}$, where $H_{\mathcal{K}}(R)=\left\{I \subseteq R_{R}: R / I \in \mathcal{K}\right\}$. The notation is taken from [4]. By the proof of Theorem 2.5 in [12], $\operatorname{Soc}_{\mathcal{K}}(R)$ is a two-sided ideal of $R$. If $\mathcal{K}=\{\operatorname{singular} R$-modules $\}$, then $\operatorname{Soc}_{\mathcal{K}}(R)$ is just the socle of $R$.

Theorem 6. Let $\mathcal{K}$ be a hereditary pre-torsion class of modules. Then every module in $\mathcal{K}$ satisfies $(P)$ if and only if either $\mathcal{K}=\{0\}$ or $S=R / \operatorname{Soc}_{\mathcal{K}}(R)$ is a division ring.

Proof. " $\Rightarrow$ ". If $0 \neq R / I_{i} \in \mathcal{K}$ for $i=1,2$, then Lemma 1 implies that $I_{1}=I_{2}$. So, either $\operatorname{Soc}_{\mathcal{K}}(R)=R$ or $\operatorname{Soc}_{\mathcal{K}}(R)$ is a maximal right ideal of $R$. Therefore, $\mathcal{K}=\{0\}$ or $S$ is a division ring.

" $\Leftarrow "$. If $\mathcal{K}=\{0\}$, then the claim follows. Suppose that $S$ is a division ring and $\mathcal{K} \neq\{0\}$. This shows that $H_{\mathcal{K}}(R)=\left\{\operatorname{Soc}_{\mathcal{K}}(R), R\right\}$. Then, for any module $M \in \mathcal{K}$ with a generating set $Y, M \cdot \operatorname{Soc}_{\mathcal{K}}(R)=0$ and thus $M$ is an $S$-module with a generating set $Y$. By Theorem $2, M_{S}=\oplus_{x \in X} x S$ for a subset $X$ of $Y$. It follows that $M_{R}=\oplus_{x \in X} x R$.

Letting $\mathcal{K}$ be the class of the singular right $R$-modules in Theorem 6 , one obtains the next corollary.

Corollary 7. Every singular $R$-module satisfies $(P)$ if and only if either $R$ is a semisimple ring or $R / \operatorname{Soc}(R)$ is a division ring.

From now on, $\mathcal{K}$ is a hereditary pre-torsion class and $\tau_{\mathcal{K}}=\left(\mathbb{T}_{\mathcal{K}}, \mathbb{F}_{\mathcal{K}}\right)$ is the torsion theory generated by $\mathcal{K}$, i.e., $\mathbb{F}_{\mathcal{K}}=\{F \in \operatorname{Mod}-R: \operatorname{Hom}(C, F)=0$ for all $C \in \mathcal{K}\}$ and $\mathbb{T}_{\mathcal{K}}=\{T \in \operatorname{Mod}-R: \operatorname{Hom}(T, F)=0$ for all $F \in \mathcal{K}\}$. By [10, Proposition 3.3], $\tau_{\mathcal{K}}$ is a hereditary torsion theory.

Theorem 8. Every module in $\mathbb{T}_{\mathcal{K}}$ satisfies $(P)$ if and only if either

(1) $\mathcal{K}=\{0\}$ or

(2) $\mathcal{K}=\mathbb{T}_{\mathcal{K}}$ and $R / \operatorname{Soc}_{\mathcal{K}}(R)$ is a division ring.

Proof. Note that $\mathcal{K}=\{0\}$ if and only if $\mathbb{T}_{\mathcal{K}}=\{0\}$. Thus the sufficiency follows from Theorem 6. For the necessity, by Theorem 6, it suffices to show that $\mathcal{K}=\mathbb{T}_{\mathcal{K}}$. If the equality does not hold, then there exists a module $M \in \mathbb{T}_{\mathcal{K}}$ but $M \notin \mathcal{K}$. Therefore, there is a cyclic submodule $x R$ of $M$ such that $x R \notin \mathcal{K}$. Since $\mathcal{K} \neq\{0\}$, there is a nonzero cyclic module $y R \in \mathcal{K}$. Then $x^{\perp} \neq y^{\perp}$. By Lemma 1 , this contradicts the assumption. So $\mathcal{K}=\mathbb{T}_{\mathcal{K}}$.

Let $\mathcal{K}$ be the class of the singular right $R$-modules. Applying Theorem 8 to $\mathcal{K}$ yields the next corollary.

Corollary 9. Every Goldie torsion module satisfies $(P)$ if and only if either $R$ a semisimple ring or $R$ is a right non-singular ring with $R / \operatorname{Soc}(R)$ being a division ring.

Acknowledgment. We thank to Prof. Yiqiang Zhou (Memorial University, Canada) for introducing us to this problem, and for his valuable comments and suggestions. 


\section{References}

[1] F. W. Anderson and K. R. Fuller, Rings and Categories of Modules (Second edition), Graduate Texts in Mathematics, 13. Springer-Verlag, New York, 1992.

[2] D. D. Anderson and J. Robeson, Bases for modules, Expo. Math. 22 (2004), no. 3, 283-296.

[3] H. Bass, Finitistic dimension and a homological generalization of semi-primary rings, Trans. Amer. Math. Soc. 95 (1960), 466-488.

[4] J. Dauns and Y. Zhou, Classes of Modules, Pure and Applied Mathematics (Boca Raton), 281. Chapman \& Hall/CRC, Boca Raton, FL, 2006.

[5] J. S. Golan, Torsion Theories, Pitman Monographs and Surveys in Pure and Applied Mathematics, 29. Longman Scientific \& Technical, Harlow; John Wiley \& Sons, Inc., New York, 1986.

[6] K. R. Goodearl, Ring Theory: Nonsingular Rings and Modules, Pure and Applied Mathematics, No. 33. Marcel Dekker, Inc., New York-Basel, 1976.

[7] J. Neggers, Cyclic rings, Rev. Un. Mat. Argentina 28 (1977), no. 2, 108-114.

[8] W. H. Rant, Minimally generated modules, Canad. Math. Bull. 23 (1980), no. 1, 103105 .

[9] L. J. Ratliff and J. C. Robson, Minimal bases for modules, Houston J. Math. 4 (1978), no. 4, 593-596

[10] B. Stenström, Rings of Quotients, Springer-Verlag, 1975.

[11] Y. Zhou, A characterization of left perfect rings, Canad. Math. Bull. 38 (1995), no. 3, $382-384$.

[12] Relative chain conditions and module classes, Comm. Algebra 25 (1997), no. $2,543-557$.

Muhammet TAmer Koşan

Department of Mathematics

FaCUlty of Science

Gebze Institute of Technology

Çayirova Campus 41400 Gebze- Kocaeli, Turkey

E-mail address: mtkosan@gyte.edu.tr

TUFAN ÖZDIN

Department of Mathematics

Faculty of Science and Literature Erzincan University

ERZINCAN, TURKEY

E-mail address: tufan.ozdin@hotmail.com 